\title{
IMPLEMENTATION OF ROTATIONAL RAMAN CHANNEL IN MULTIWAVELENGTH AEROSOL LIDAR TO IMPROVE MEASUREMENTS OF PARTICLE EXTINCTION AND BACKSCATTERING AT 532 NM
}

\author{
Igor Veselovskii ${ }^{1}$, David N. Whiteman ${ }^{2}$, Michael Korenskiy ${ }^{1}$, A.Suvorina ${ }^{1}$, Daniel Perez-Ramirez ${ }^{2,3}$ \\ ${ }^{1}$ Physics Instrumentation Center, Troitsk, Moscow Region, 142190, Russia, E-mail: \\ E-mail: igorv@pic.troitsk.ru \\ ${ }^{2}$ Mesoscale Atmospheric Processes Laboratory, NASA Goddard Space Flight Center, Greenbelt, MD 20771, USA, \\ E-mail: david.n.whiteman@nasa.gov \\ ${ }^{3}$ Applied Physics Department, University of Granada, 18071, Granada, Spain.
}

\begin{abstract}
We describe a practical implementation of rotational Raman (RR) measurements in an existing MieRaman lidar to obtain measurements of aerosol extinction and backscattering at $532 \mathrm{~nm}$. A $2.3 \mathrm{~nm}$ width interference filter was used to select a spectral range characterized by low temperature sensitivity within the anti-Stokes branch of the RR spectrum. Simulations demonstrate that the temperature dependence of the scattering cross section does not exceed $1.0 \%$ in the $230-300 \mathrm{~K}$ range making accurate correction for this dependence quite easy. With this upgrade, the NASA/GSFC multiwavelength Raman lidar has demonstrated useful $\alpha_{532}$ measurements and was used for regular observations. Examples of lidar measurements and inversion of optical data to the particle microphysics will be given in presentation.
\end{abstract}

\section{INTRODUCTION}

Mie-Raman lidars acquiring elastic backscatters simultaneously with nitrogen vibrational Raman signal at $387 \mathrm{~nm}$ and $608 \mathrm{~nm}$ are widely used for independent mesurements of particle backscattering $(\beta)$ and extinction $(\alpha)$ coefficients. While high signalto-noise measurements of nitrogen vibrational scattering at $387 \mathrm{~nm}$ during both daytime and nighttime have been possible, the use of vibrational Raman scattering at $608 \mathrm{~nm}$ has posed certain problems. First of all, cross section of vibrational Raman scattering is almost three orders lower comparing to Raleigh one, which limits range and temporal resolution of the measurements. Another issue related to Raman nitrogen measurements at both $387 \mathrm{~nm}$ and $608 \mathrm{~nm}$ is the significant wavelength shift of the nitrogen Raman component with respect to the laser line. As a result, information about the spectral dependence of aerosol extinction is needed when $\alpha$ and $\beta$ are computed [1], which can be an additional source of uncertainty. A well-known solution for both of these issues is the use of High
Spectral Resolution Lidar (HSRL). The recent results provided by the NASA/LaRC airborne lidar demonstrate the possibility of reliable HSRL operation at both $355 \mathrm{~nm}$ and $532 \mathrm{~nm}$ [2]. However such lidars, due to their complexity and cost, are still unique instruments. At the same time, there are numerous existing Raman lidars which can potentially be upgraded to improve Raman measurements at $608 \mathrm{~nm}$. One of the ways this can be accomplished is to use pure rotational Raman (RR) scattering instead vibrational scattering. The small spectral separation of the elastic and RR components of the signal implies that aerosol backscatter and extinction can be obtained without information about the Ångström exponent. Furthermore, the backscatter cross section of anti-Stokes component of pure RR nitrogen scattering exceeds that of vibrational scattering by approximately a factor of 15-20, when all lines for nitrogen and oxygen are integrated [3].

Pure rotational Raman scattering is widely used in laser remote sensing for the measurement of atmospheric temperature [e.g. 4,5], where the pure $\mathrm{RR}$ and elastic signals are generally separated by the use of narrow-band interference filters or diffractiongrating- spectrometers. Rotational Raman scattering can also be used for the particle extinction and backscattering calculation. However, an important consideration in the use of RR measurements for calculating $\alpha$ and $\beta$ is the temperature dependence of the RR spectra of $\mathrm{N}_{2}$ and $\mathrm{O}_{2}$ molecules. This issue in principle can be resolved by using the sum of lidar returns from the Stokes and anti-Stokes branches, which is essentially temperature independent. However, measuring all Stokes and anti-Stokes lines with equal efficiency is technologically difficult. The alternate approach considered in this paper is to select a portion of the RR spectrum characterized by a low temperature sensitivity inside one of the spectral branches. Such an approach simplifies the implementation of a RR channel in conventional 
multiwavelength Raman lidar systems without significant modification of the receiver design. In the approach presented here, selection of the RR lines is done by use of a wide-band interference filter which transmits the desired portion of the anti-Stokes branch. Centering the filter transmission band near the temperature insensitive lines of the RR spectrum decreases the temperature sensitivity of the RR scattering cross section to a level appropriate for tropospheric measurements.

\section{NUMERICAL SIMULATIONS}

Fig.1 shows RR spectra of nitrogen and oxygen weighted with corresponding number density fractions at $\mathrm{T}=270 \mathrm{~K}$ for a laser wavelength of 532.12 $\mathrm{nm}$. In our implementation, the filter transmitted only portions of the anti-Stokes branch in order to have the possibility to shift the filter transmission band toward shorter wavelengths by filter tilting, if necessary. The differential cross section of RR scattering can be calculated as:

$\sigma^{R R}=\eta_{N_{2}} \sum_{i} \xi_{R}\left(\lambda_{i}\right) \sigma_{N_{2}}^{R R}\left(\lambda_{i}\right)+\eta_{O_{2}} \sum_{k} \xi_{R}\left(\lambda_{k}\right) \sigma_{o_{2}}^{R R}\left(\lambda_{k}\right)$

Here $\sigma_{N_{2}}^{R R}, \sigma_{O_{2}}^{R R}$, are the differential scattering cross sections of nitrogen and oxygen, $\xi_{R}\left(\lambda_{i}\right)$ are the filter transmittances of the Raman channel at wavelengths $\lambda_{\mathrm{i}}$ corresponding the lines of RR spectrum, $\eta_{N_{2}}, \eta_{O_{2}}$ are the fractions of nitrogen and oxygen concentration.

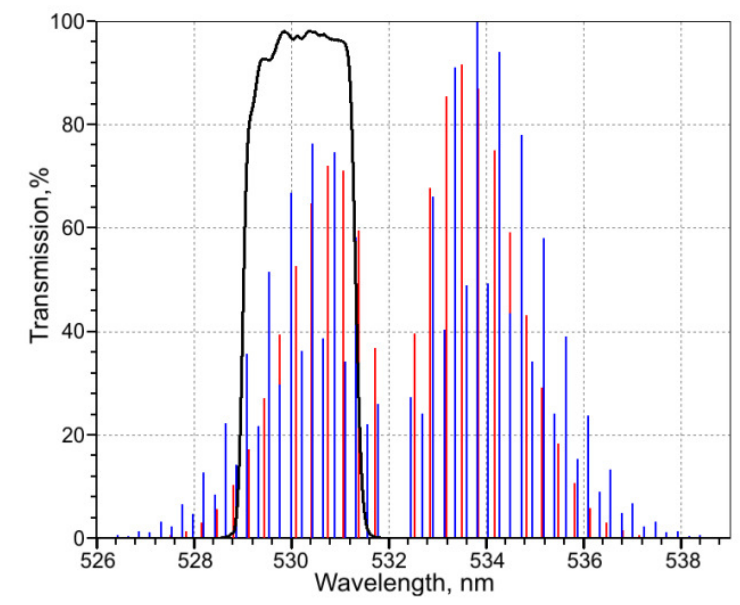

Fig.1. Spectrum of RR scattering by nitrogen (blue) and oxygen (red) for $\mathrm{T}=300 \mathrm{~K}$. Black line shows transmittance of the interference filter used in the experiment. Laser line is at $532.12 \mathrm{~nm}$.

The frequency shift of rotational lines with respect to the frequency of the laser radiation is small compared with vibrational scattering, so in practice the spectral dependence of particle extinction can be neglected. The intensities of the individual lines in the RR spectrum have a strong dependence on temperature, so the scattering cross section is temperature (and thus range) dependent. For $\mathrm{RR}$ the standard expressions for calculation of extinction and backscattering coefficients [1] is modified as:

$$
\begin{aligned}
& \alpha^{a}(z)=\frac{1}{2} \frac{d}{d z} \ln \left[\frac{N(z)}{P_{R}(z) z^{2}}\right]-\alpha^{m}(z)+\Delta \alpha^{T} \\
& \beta^{a}(z)=-\beta^{m}(z)+\beta^{m}\left(z_{0}\right) \cdot \frac{P_{R}\left(z_{0}\right) P_{L}(z) N(z)}{P_{L}\left(z_{0}\right) P_{R}(z) N\left(z_{0}\right)} F(z)
\end{aligned}
$$

Where $P_{L, R}(z)$ is the power of elastic and Raman backscatters, $N(z)$ is the density of air molecules. The reference height $\mathrm{z}_{0}$ is chosen in the free troposphere where $\beta_{L}^{a}\left(z_{0}\right)<<\beta_{L}^{m}\left(z_{0}\right)$. Indices "a" and " $\mathrm{m}$ " correspond to aerosol and molecular components of backscattering and extinction coefficients.

$\beta^{T}=\beta^{a}+\beta^{m}, \quad \alpha^{T}=\alpha^{a}+\alpha^{m}$

The expression for extinction (2) contains the term $\Delta \alpha^{T}=\frac{1}{2} \frac{d}{d z} \ln \left[\sigma^{R R}(z)\right]$ arising from the height variation of atmospheric temperature and, as a result, variation of the scattering cross section. This term can be significant in the presence of strong temperature gradients.

The expression (3) for backscattering coefficient contains the factor $F(z)=\frac{\sigma_{R R}(z)}{\sigma_{R R}\left(z_{0}\right)}$ responsible for the temperature dependence of $\beta^{a}$, arising from temperature variation from $\mathrm{T}_{0}$ to $\mathrm{T}$ at heights $\mathrm{z}_{0}$ and $\mathrm{z}$ respectively. The error in backscattering $\varepsilon_{\beta}^{T}$ due to neglecting of this temperature dependence $(F=1)$ can be estimated from (3) as:

$\varepsilon_{\beta}^{T}=\frac{\beta^{a}-\beta_{F=1}^{a}}{\beta^{a}}=\frac{\beta^{T}}{\beta^{T}-\beta^{m}} \frac{F-1}{F}=\frac{R}{R-1} \frac{F-1}{F}$

where $\mathrm{R}$ is the scattering ratio $\frac{\beta^{T}}{\beta^{m}}$. For $\beta^{a}>>\beta^{m}$ the error $\varepsilon_{\beta}^{T}$ becomes $\frac{F-1}{F}$, while for low aerosol loading there is an enhancement factor $\frac{R}{R-1}$.

To quantify the possible uncertainties in the evaluation of backscattering and extinction coefficients arising from the temperature dependence of $\sigma^{R R}$, numerical simulation studies were performed. The RR line with least temperature sensitivity is in the vicinity $530.4 \mathrm{~nm}(\mathrm{~J}=9)$ for $\mathrm{O}_{2}$ and in vicinity of $530.2 \mathrm{~nm}(\mathrm{~J}=7)$ for $\mathrm{N}_{2}$, so centering the filter transmission band near these values will 
minimize the sensitivity of $\sigma^{R R}$ to changes in temperature. When choosing filter parameters the desire is to maximize the cross section $\sigma^{R R}$ by including only the strongest rotational lines since unnecessarily wide transmission band will lead to an increase of the sky background noise and an overall decrease in signal to noise under daytime conditions.

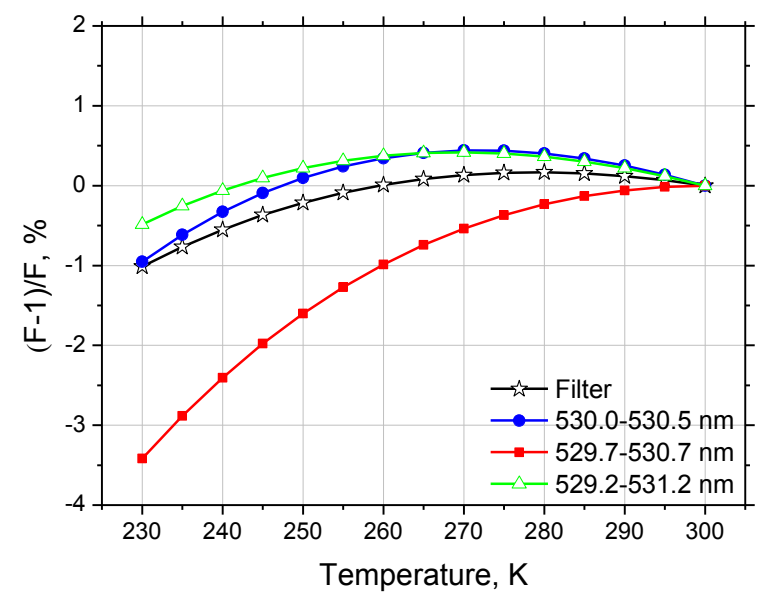

Fig.2. The relative change of RR scattering cross section $(\mathrm{F}-1) / \mathrm{F}$ as a function of temperature for spectral range $530.0-530.5 \mathrm{~nm}, 529.7-530.7 \mathrm{~nm}, 529.2-531.2 \mathrm{~nm}$. Open stars show results for the interference filter used in the experiment.

Fig.2 shows the change of $\frac{F-1}{F}$ as a function of temperature for the spectral intervals of Raman spectrum 530.0-530.5 nm, 529.7 - 530.7 nm and 529.2-531.2 nm. Computations were performed with $\mathrm{T}$ varying in the range $230-300 \mathrm{~K}$ where the reference temperature $T_{0}$ was taken to be $T_{0}=300 K$. For all intervals the relative change of RR scattering cross section $\frac{F-1}{F}=\frac{\sigma^{R R}(T)-\sigma^{R R}\left(T_{0}\right)}{\sigma^{R R}(T)}$ does not exceed $3.5 \%$. The ratios of $\sigma^{R R}\left(T_{0}\right)$ to the total cross section of anti-Stokes branch for these three intervals are 0.2 , 0.37 and 0.7. Of these intervals, that of 529.2-531.2 $\mathrm{nm}$ is the most attractive, as it contains approximately $70 \%$ of the Raman signal provided by the entire antiStokes band and possesses a small temperature dependence (the variation of $\sigma^{R R}$ is below $1.0 \%$ ). Based on these considerations, we chose this interval as the target for the interference filter design.

The transmission spectrum of the interference filter used in our experiments, taken from the manufacturer's data sheet (Alluxa, CA, USA), is shown in fig.1. The filter has transmission greater than $95 \%$ at the peak with bandwidth of approximately $2.3 \mathrm{~nm}$ FWHM. The suppression of the elastic scattering at $532.12 \mathrm{~nm}$ is performed with
OD $>4$. The variation of $\frac{F-1}{F}$ for this filter in 230-

$300 \mathrm{~K}$ temperature range doesn't exceed $1 \%$, so in many practical cases $F=1$ in (3) can be used.

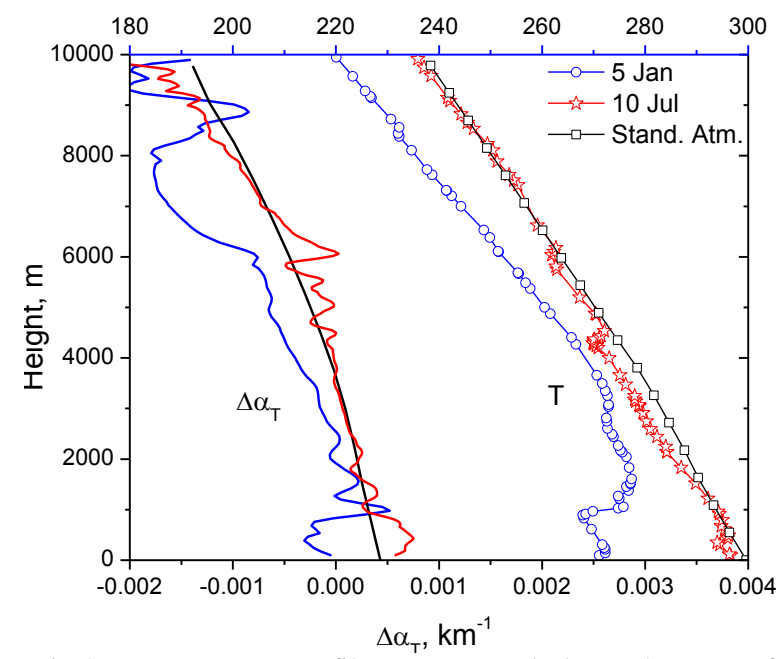

Fig.3. Temperature profiles (open symbols) and errors of extinction coefficient calculation $\Delta \alpha_{\mathrm{T}}$ due to height variation of temperature (solid lines). Computations were performed for US Standard Atmosphere temperature profile (black) and for temperature measured by radiosonde on 5 January (blue) and 10 July 2013 (red).

The error of the extinction coefficient calculation $\Delta \alpha_{T}$ induced by the temperature height variation depends, as it follows from (2), on the gradient of the temperature profile. To estimate this error we have tested the temperature profiles from the US Standard Atmosphere model and from radiosonde launches performed at Dulles airport, VA in the vicinity of Washington DC. The results of computations are shown in fig.3. To calculate the range derivation from discrete temperature measurements the cubic spline over 7 points was used. For the model temperature profile the decrease of the temperature with height provides a negative contribution to the calculated particle extinction. The error $\Delta \alpha_{T}$ changes with height but it is below $0.002 \mathrm{~km}^{-1}$ for the heights up to $10 \mathrm{~km}$. The same figure shows the results for radiosonde launches on 5 January and 10 July 2013. Corresponding temperature profiles present low-scale oscillations, still for both days $\Delta \alpha_{T}<0.002 \mathrm{~km}^{-1}$. Computations performed for days at different seasons have demonstrated that, in general, temperature variations contribute an error in calculated extinction of less than $2 \%$ for extinction values of $0.1 \mathrm{~km}^{-1}$ and greater.

Using the scattering cross sections provided by Fenner [3] we estimate that the scattered power in the RR channel is a factor of 15 higher than that of vibrational nitrogen Raman channel at $608 \mathrm{~nm}$. Thus, 
under skylight limited conditions, the RR channel would provide greater signal-to-noise if the width of the filter used for the vibrational Raman measurement is greater than approximately $0.15 \mathrm{~nm}$..

\section{MODIFICATION OF GSFC RAMAN LIDAR}

A description of the NASA/GSFC multiwavelengh Raman lidar is presented in [6]. To incorporate the $\mathrm{RR}$ channel at $530 \mathrm{~nm}$ the lidar receiving module was modified. The optical configuration permitted simultaneous measurements of the vibrational and rotational Raman signals. The spectral components at $608 \mathrm{~nm}$ and $532 \mathrm{~nm}$ were separated by a dichroic mirror. The additional beamsplitter then reflected approximately $10 \%$ portion of the 532 signal into the Mie channel with more than $85 \%$ being transmitted into the RR channel. Two RR filters each possessing the characteristics shown in fig. 1 were used together to provide more than 8 orders of magnitude rejection of the elastic signal. To compare the scattered power in $608 \mathrm{~nm}$ and RR $530 \mathrm{~nm}$ channels, we made two sequential measurements using the same R1924 PMT for both channels. After correction for difference in quantum efficiency of the photocathode at $608 \mathrm{~nm}$ and $530 \mathrm{~nm}$, we estimate the enhancement of scattered power in RR channel compared with the vibrational one to be more than a factor 10 .

The initial measurements were taken under cloudy conditions to test the blocking of the Mie scattering by the RR filters. Those tests did not reveal any enhancement of the signal measured in the RR channel due to backscatter from the base of the cloud. Fig.4 shows backscattering and extinction coefficients at $532 \mathrm{~nm}$ calculated from vibrational and rotational Raman signals. The maximal difference between backscattering profiles was observed in the maximum of scattering layer at approximately $1750 \mathrm{~m}$ height and did not exceed $2 \%$.This figure also clearly demonstrates the improvement of the extinction calculation when the RR signal at $530 \mathrm{~nm}$ is used instead of that of vibrational scattering at $608 \mathrm{~nm}$.

\section{CONCLUSION}

The use of rotational Raman scattering, instead of vibrational, removes the concern due to wavelength scaling, while simultaneously permitting measurements to take advantage of a much larger cross section. We have presented a practical approach to implementing rotational Raman measurements in multiwavelength aerosol lidar using sharp edge interference filters to separate the RR and Mie signals. During the design phase of the development, numerical simulations were performed demonstrating that a measurement of aerosol extinction could be made with low temperature dependence using a portion of the either anti-Stokes of Stokes components. This selection also achieved a signal increase of more than a factor or 10 when compared with vibrational nitrogen. Upgraded this way the NASA/GSFC multi-wavelength Raman lidar has demonstrated improved capabilities for $\alpha_{532}$ measurements which in turn has improved the inversion of $3 \beta+2 \alpha$ optical data to particle microphysics. The examples of regular lidar observations will be given in presentation.

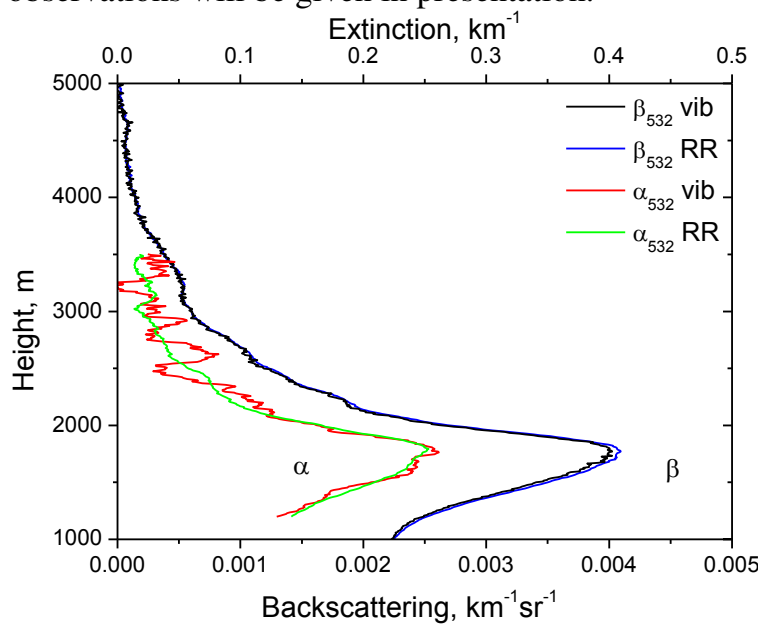

Fig.4. Extinction and backscattering coefficients at $532 \mathrm{~nm}$ computed from nitrogen vibrational Raman and from RR signal

\section{References}

[1]. Ansmann, A., et al.: Combined Raman elasticbackscatter lidar for vertical profiling of moisture, aerosols extinction, backscatter, and lidar ratio, Appl.Phys.B, 55, 18-28, 1992.

[2] Müller, D., et al.: Airborne Multiwavelength High Spectral Resolution Lidar (HSRL-2) observations during TCAP 2012: vertical profiles of optical and microphysical properties of a smoke/urban haze plume over the northeastern coast of the US, Atmos. Meas. Tech., 7, 3487-3496, 2014.

[3] Fenner, W. R., et al.: Raman cross section of some simple gases, J.Opt.Soc.Amer. 63, 73-77, 1973. [4] Behrendt, A. and Reichardt, J.: Atmospheric temperature profiling in the presence of clouds with a pure rotational Raman lidar by use of an interferencefilter-based polychromator, Appl. Optics, 39, 13721378, 2000.

[5] Arshinov,Y., et al.: Daytime operation of a pure rotational Raman lidar by use of a Fabry-Perot interferometer, Appl.Opt. 44, 3593-3603, 2005.

[6] I. Veselovskii, et al. "Characterization of forest fire smoke event near Washington, D.C. in Summer 2013 with multi-wavelength lidar", Atmos. Chem. Phys. 15, 1647-1660, 2015. 